\title{
SYNTHESIS AND LUMINESCENCE OF
}

\author{
SOLUBLE meso-UNSUBSTITUTED
}

\section{TETRABENZO- AND TETRANAPHTHO}

\section{$[2,3]$ PORPHYRINS}

\section{SUPPORTING INFORMATION}

\author{
Olga S. Finikova, ${ }^{a}$ Andrei V. Cheprakov ${ }^{b}$ and Sergei A. Vinogradov ${ }^{a}$ *
}

${ }^{a}$ Department of Biochemistry and Biophysics, University of Pennsylvania, Philadelphia,

PA 19104; ${ }^{b}$ Department of Chemistry, Moscow State University, Moscow 119899 , Russia.

* To whom correspondence should be addressed. Phone: (215)-898-6382, FAX: (215)573-3787, E-mail: vinograd@mail.med.upenn.edu 


\section{Table of Contents}

Materials and Methods

References and Notes S3

NMR spectra S4 


\section{Materials and Methods}

All solvents were purified according to the standard procedures. Selecto silica gel or neutral aluminum oxide (Brockmann I, 150 mesh) was used for column chromatography. All starting materials and reagents were obtained from standard commercial suppliers. Analytical TCL's were run on commercial Riedel-de Haën plates coated with silica gel $60 \mathrm{~F}_{264}(0.2-\mathrm{mm}$ thickness).

Static fluorescence and phosphorescence measurements were performed on SPEX Fluorolog-2 spectrofluorometer (Jobin-Yvon Horiba, Inc.), equipped with an infra-red enhanced R2658P PMT (Hamamatsu). Phosphorescence lifetimes were measured using an in-house constructed frequency domain phosphorometer. ${ }^{1}$ Solutions for phosphorescence measurements were purged with Ar. The absorbances of the samples at the wavelengths used for excitation were kept below 0.05 OD. The quantum yields were obtained by computing the integrals of the corrected emission spectra and referencing them to the fluorescence quantum yield of ZnTPP in deoxygenated benzene $\left(\phi_{\text {fluor }}=0.033\right) .^{2}$ The spectra were normalized by the optical density of the samples at the excitation wavelengths, relative photon intensity of the source and quantum efficiency of the detector throughout the emission range. previously. $^{3}$

Phosphorescence oxygen quenching constants $\left(\mathrm{K}_{\mathrm{q}}\right)$ were determined as described

\section{References and Notes}

(1) Vinogradov, S. A.; Fernandez-Seara, M. A.; Dugan, B. W.; Wilson, D. F. Rev. Sci. Instrum. 2001, 72, 3396.

(2) Quimby, D. J.; Longo, F. R. J. Am. Chem. Soc. 1975, 97, 5111.

(3) Rozhkov, V.V.; Wilson, D.F.; Vinogradov, S.A. Macromolecules 2002, 35, 1991. 
NMR spectra 


\title{
2b, 1H NMR, CDCl3
}

3.00

\section{5}

\begin{abstract}
4
\end{abstract}

\begin{abstract}
3
\end{abstract} OF1: 2797.3

NA: 8

LB: 0.0

F1: 499.836

EX: s2pul

F2: 499.836

a de
PTS1d: 16384 


\section{2b, 13C NMR, CDCI3}

OF1: 11943.5

PD: 2.0 sec PTS1d: 8192

LB: 0.0

WinNuts - \$of_tbpSO2_13C.fid 


\section{3b, 1H NMR, CDCl3}

5.09

6.22

NA: 8

4.18 4.00 


\section{3b, 13C NMR, CDCl3}

F1: 125.695 F2: 499.836
OF1: 11940.2

NA: 2832 PTS1d: 8192

LB: 0.0

WinNuts - \$of_3b.fid 
3c, 1H NMR, CDCl3

1.00 


\section{3c, 13C NMR, CDCl3}

160

140

120

100

80

60

OF1: 11939.3

NA: 128

SW1: 25000

PW: 13.0 usec

PD: $2.0 \mathrm{sec}$

LB: 0.0

F2: 499.842

EX: s2pul

\begin{tabular}{|c|} 
PTS1d: 8192 \\
WinNuts - \$of96_13C.fid
\end{tabular}


4b, 1H NMR, CDCl3 


\section{4b, 13C NMR, CDCl3}

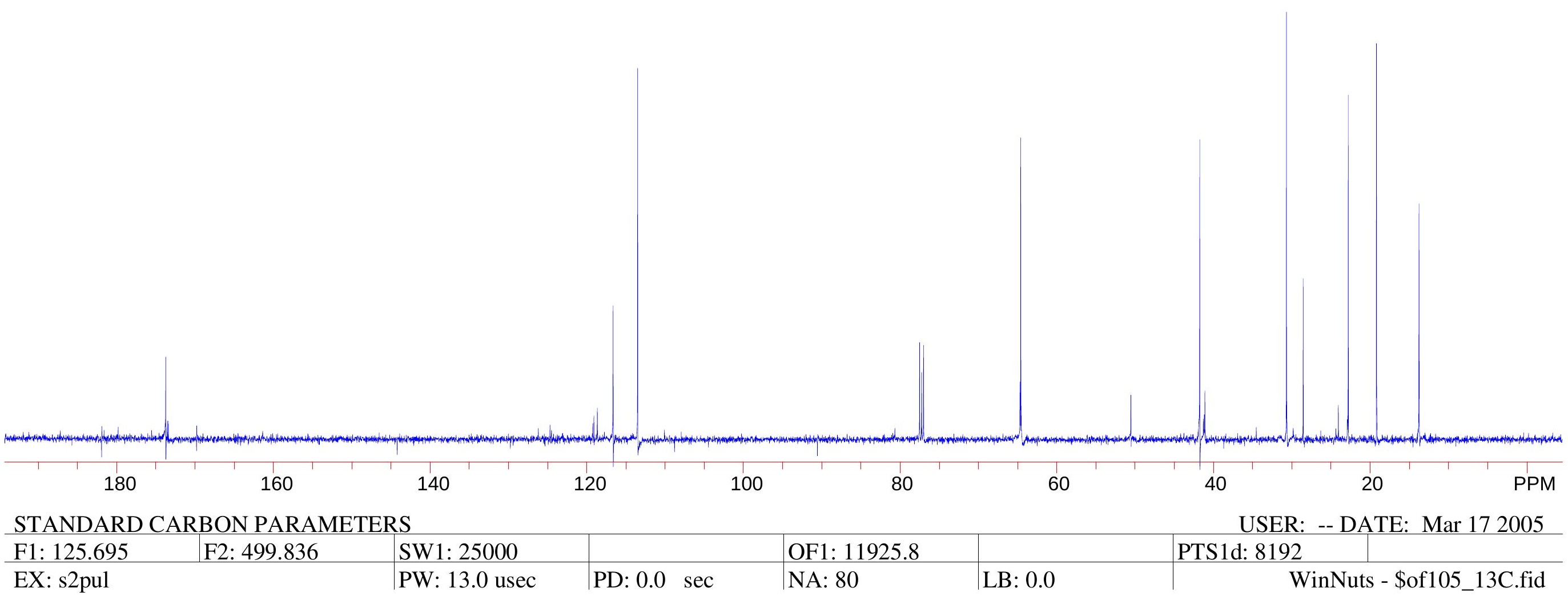




\section{$5 a$ (mixture of isomers), $1 \mathrm{H} \mathrm{NMR,} \mathrm{CDCl} 3$}

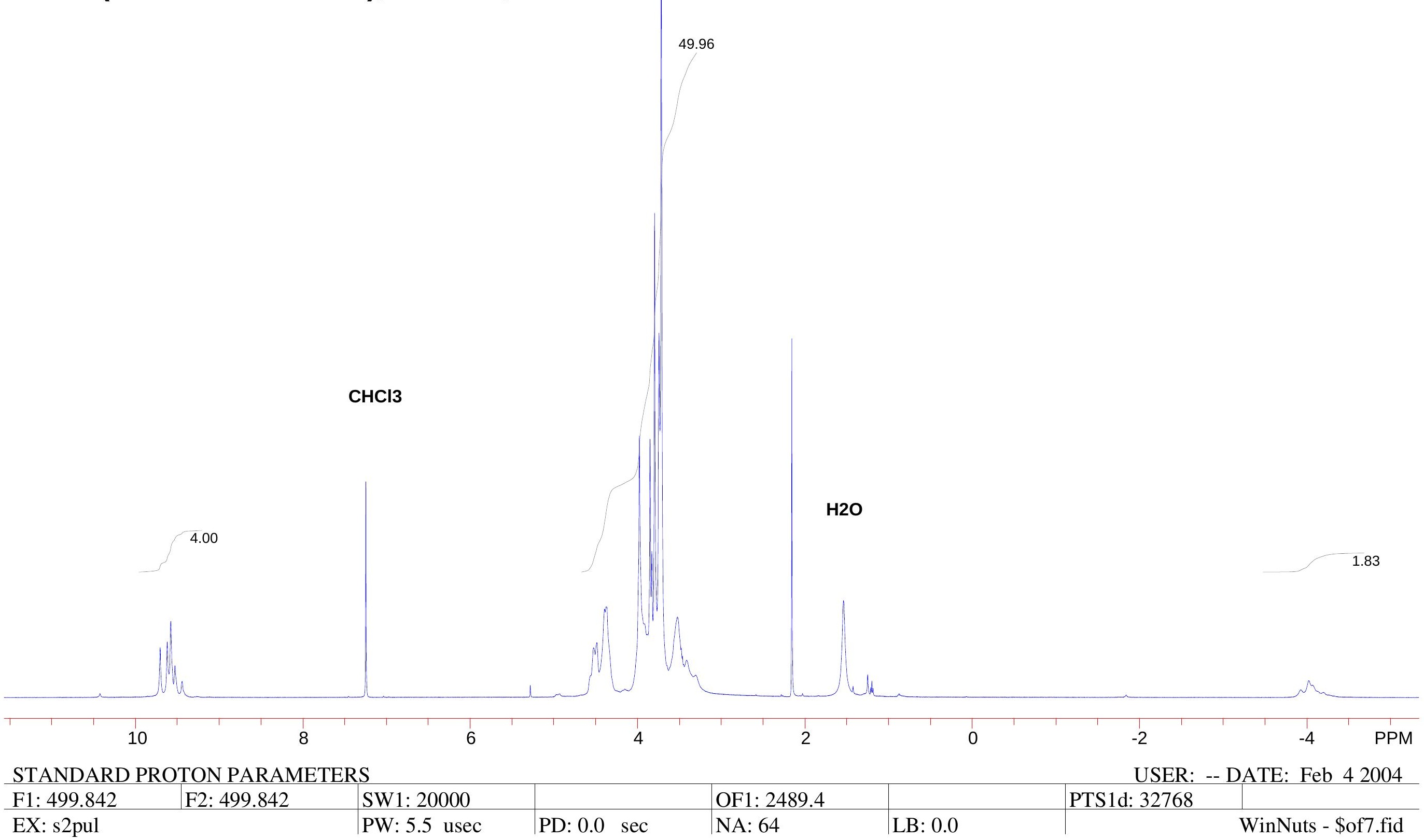




\section{S14}

Zn-5a (mixture of isomers), 1H NMR, DMSO-d6 
$5 b$ (mixture of isomers), $1 \mathrm{H} \mathrm{NMR,} \mathrm{CDCl} 3$

41.81

OF1: 2491.9

NA: 8
SW1: 20000

PW: 5.5 usec
F1: 499.842 EX: s2pul

\begin{abstract}
F2: 499.842
\end{abstract}
PD: 0.0 sec

PD: $0.0 \mathrm{sec}$
USER: -- DATE: May 142004 PTS1d: 32768

WinNuts - \$of36tchp.fid 
Zn-5b (mixture of isomers), 1H NMR, DMSO-d6

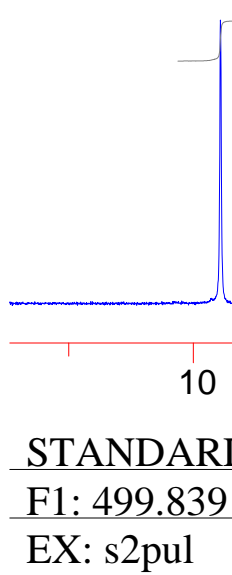

OF1: 2977.5

NA: 8
F2: 499.836

PD: 1.0 sec

LB: 0.0

2

USER: -- DATE: Mar 312005 PTS1d: 16384

WinNuts - \$of_buzn.fid 


\section{6b, 1H NMR, CDCl3}

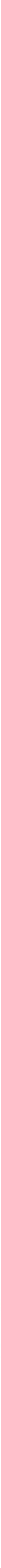




\section{6b (dication form) in TFA-d}

tfa

tfa 

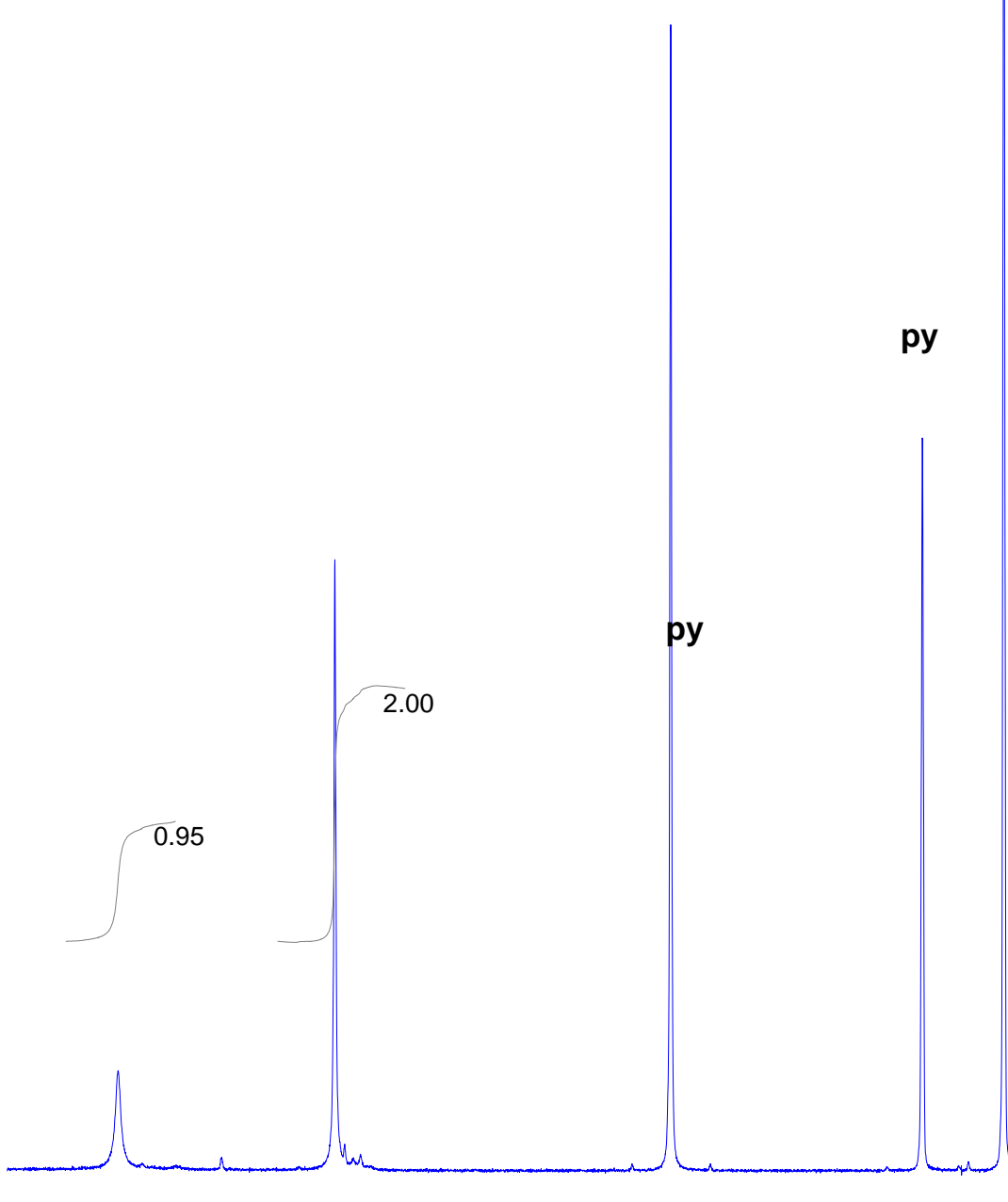

py

\section{Zn-6b, 1H NMR, pyridine-d5 (50 $\left.{ }^{\circ} \mathrm{C}\right)$}

10

6

4

2

PPM

STANDARD 1H OBSERVE F1: 499.835 EX: s2pul
SW1: 8000 PW: 11.0 usec
OF1: 2804.9

NA: 8

USER: -- DATE: Dec 202004 PTS1d: 16384

WinNuts - \$of78_py50.fid 


\section{Zn-6b, 13C NMR, pyridine-d5 (50 \&)}




\section{Pd-6b, 1H NMR, pyridine-d5 (70 $\mathrm{C})$}

USER: -- DATE: May 242004 PTS1d: 32768

WinNuts - \$of49_py70.fid 
F1: 125.697 F2: 499.842 PTS1d: 8192 
H2O 


\section{S24}

Pt-6b, 13C NMR, pyridine-d5 (70 $\left.{ }^{\circ} \mathrm{C}\right)$

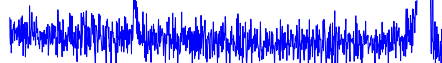

160

140

120

100

60

40

20

PPM

STANDARD CARBON PARAMETERS

F1: 125.695

EX: s2pul F2: 499.836

SW1: 25000

PW: 13.0 usec
OF1: 12193.0

NA: 17382

USER: -- DATE: Dec 232004 PTS1d: 8192

WinNuts - \$ofPt_13C.fid 


\section{Fe-6b, 1H NMR, pyridine-d5}

6.04

py

py

1.00

$$
10
$$

$$
8
$$

6

4

2

$4.53 \quad 4.51$

USER: -- DATE: Nov 172004 EX: s2pul 


\section{Fe-6b, 13C NMR, pyridine-d5}
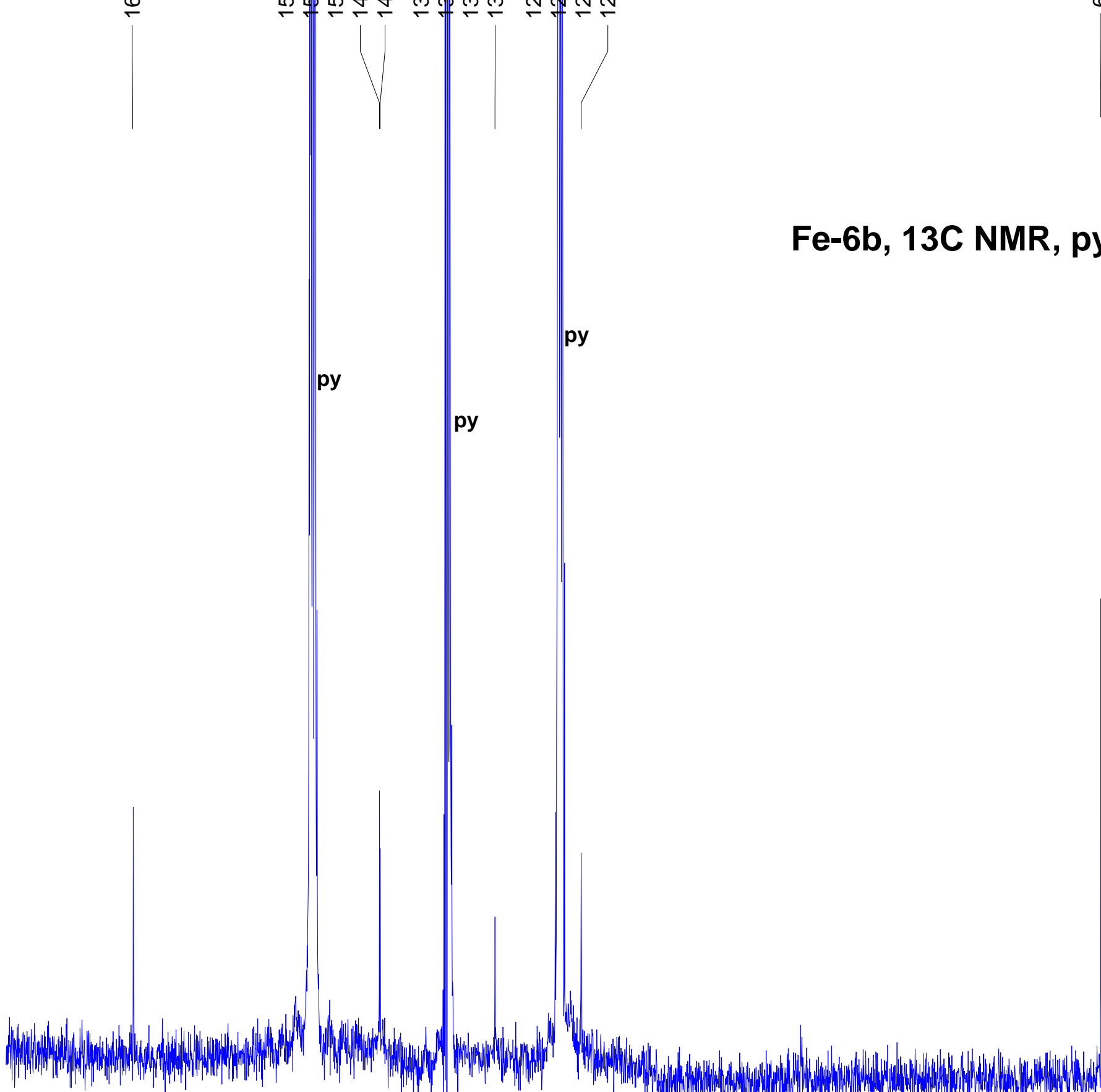

$\stackrel{\Omega}{\Omega}$

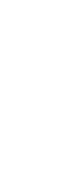

$180160 \quad 140$

STANDARD CARBON PARAMETERS

F1: 125.695

EX: s2pul

F2: 499.836

SW1: 34996

PW: 13.0 usec

120

100

80

60

OF1: 12000.6

PD: 2.0 sec

NA: 17344

LB: 0.0 PTS1d: 8192

USER: -- DATE: Dec 212004 WinNuts - \$of_Fe_13C.fid 


\section{8b, 1H NMR, CDCl3}

OF1: 2490.1

NA: 8 


\section{8b, 13C NMR, CDCl3}

100

80

60

40

STANDARD CARBON PARAMETERS

F1: 125.695 F2: 499.836 SW1: 25000 PW: 13.0 usec
OF1: 11937.3

NA: 256

USER: -- DATE: Mar 212005 PTS1d: 8192 


\section{8c, 1H NMR, CDCl3}

STANDARD PROTON PARAMETERS F1: 499.842 EX: s2pul

\begin{abstract}
F2: 499.842
\end{abstract}
SW1: 20000

PW: 5.5 usec OF1: 2491.3 NA: 8 PTS1d: 32768 


\section{8c, 13C NMR, CDCl3}

STANDARD CARBON PARAMETERS

F1: 125.697

EX: s2pul

\begin{abstract}
F2: 499.842
\end{abstract}
SW1: 25000

PW: 13.0 usec
120
100
80

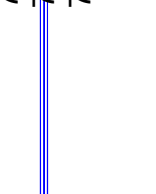




\section{9b, 1H NMR, CDCl3}

OF1: 2492.1

PD: $0.0 \mathrm{sec}$

NA: 8
3 


\section{9b, 13C NMR, CDCl3}

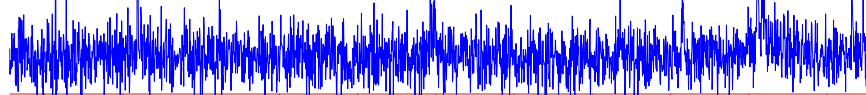

160

140

120

100

80

60

40

20

STANDARD CARBON PARAMETERS

F1: 125.695 F2: 499.836

SW1: 25000

PW: 13.0 usec
OF1: 11935.8

NA: 432

USER: -- DATE: Mar 212005 PTS1d: 8192 
吕

ㄱำ

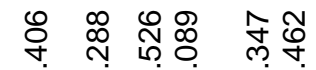

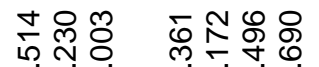

콬코융소

ヘト̃

लें लिं

S34

\section{9c, 13C NMR, CDCl3}

80

60

40 USER

STANDAR
F1: 125.695

EX: s2pul

SW1: 25000

PW: 13.0 usec
OF1: 11945.6

NA: 896 PTS1d: 8192

PD: $2.0 \mathrm{sec}$

WinNuts - \$of74_13C.fid 
10b, 1H NMR, CDCl3

$\mathrm{CHCl3}$

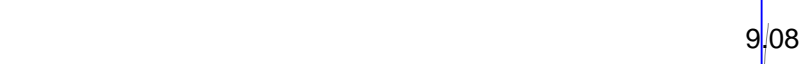

2.00

2.03

6.26

9.08

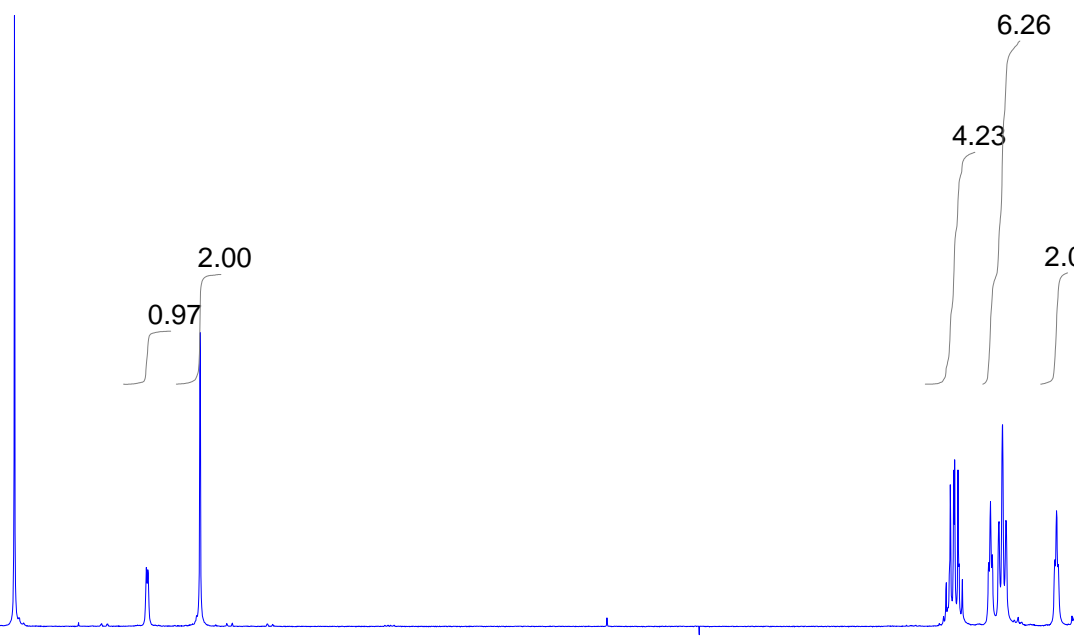

7

6

5

4

3

2

1

PPM

STANDARD PROTON PARAMETERS

F1: 499.842

SW1: 20000

OF1: 2491.9

USER: -- DATE: Sep 162004

EX: s2pul

PW: 5.5 usec

PD: 0.0 sec

NA: 66

LB: 0.0

PTS1d: 32768

WinNuts - \$of79_1c.fid 
甩离

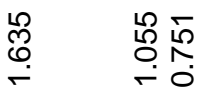

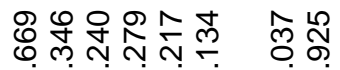

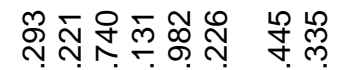

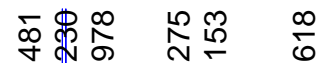

रํํㅇ

10b, 13C NMR, CDCl3

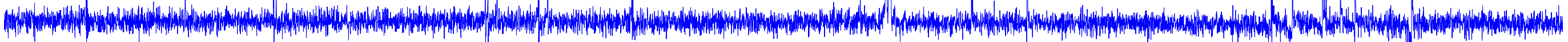

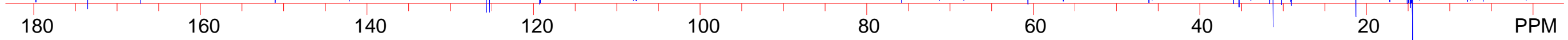

STANDARD CARBON PARAMETERS

F1: 125.697

F2: 499.842

SW1: 25000

OF1: 11940.4

USER

PW: 13.0 usec

PD: 2.0 sec

NA: 2302

LB: 0.0

WinNuts - \$of79c_13C.fid 
ำ 옹

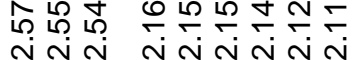

11b, 1H NMR, CDCI3 


\section{1b, 13C NMR, CDCl3}

F1: 125.697 F2: 499.842 USER: -- DATE: Sep 162004 EX: s2pul PW: 13.0 usec OF1: 11943.3

NA: 2050

LB: 0.0

WinNuts - \$of80_13C.fid PTS1d: 8192 
13b, 1H NMR, solvent mixture pyridine-d5 - PhNO2-d5 (1:1)

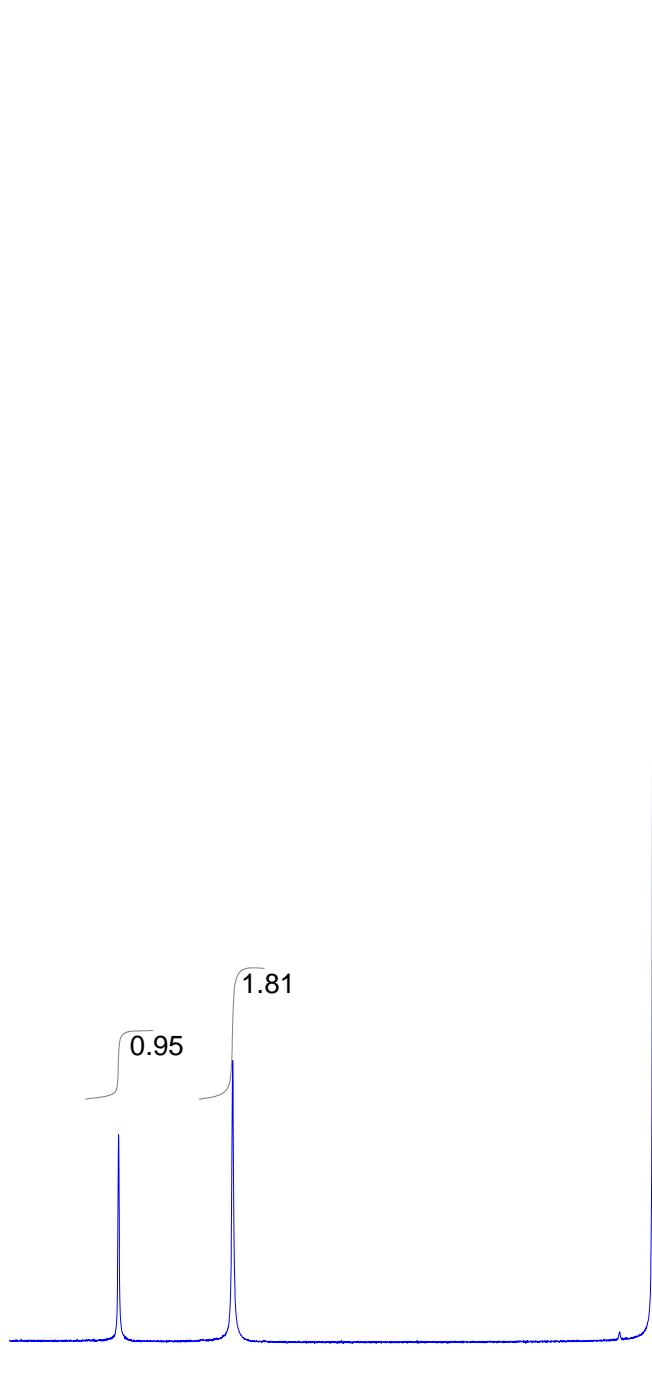

10

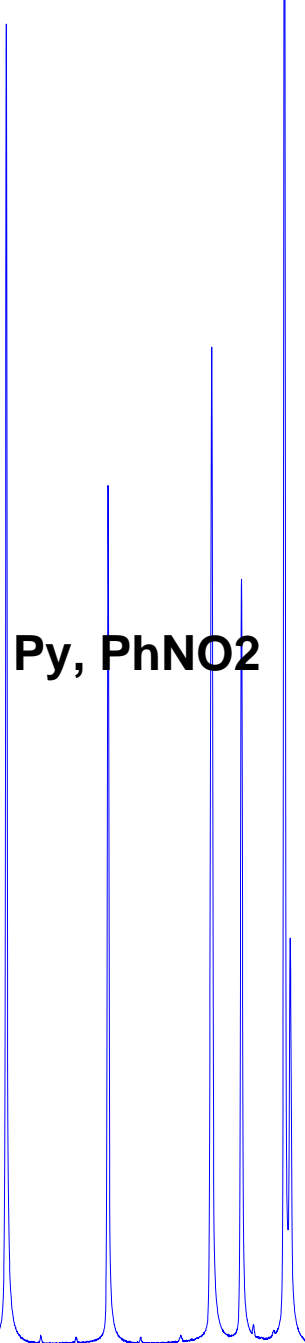

8

6

SW1: 8000

PW: 11.0 usec
8.29

4.08

4

2

0

PPM USER: -- DATE: Apr 142005 PTS1d: 16384

OF1: 2808.9

PD: 1.0 sec

NA: 160

LB: 0.0

WinNuts - \$of1i.fid 
13b, 13C NMR, solvent mixture pyridine-d5 - PhNO2-d5 (80 $\mathrm{C})$

160

140

120

100

80

60

40 USER

SW1: 25000

PW: 13.0 usec
OF1: 12035.8

NA: 16928
PD: $2.0 \mathrm{sec}$

\begin{abstract}
F2: 499.836
\end{abstract}
F1: 125.68
EX: s2pul

WinNuts - \$of1i_13C.fid 


\section{Zn-13b, 1H NMR, pyridine-d5}

1.81

0.96 


\section{Zn-13b, 13C NMR, pyridine-d5}

\begin{abstract}
F2: 499.836
\end{abstract}
SW1: 25000

PW: 13.0 usec
OF1: 12187.7

NA: 15168

USER: -- DATE: Apr 122005 PTS1d: 8192 
OF1: 2065.4

NA: 64
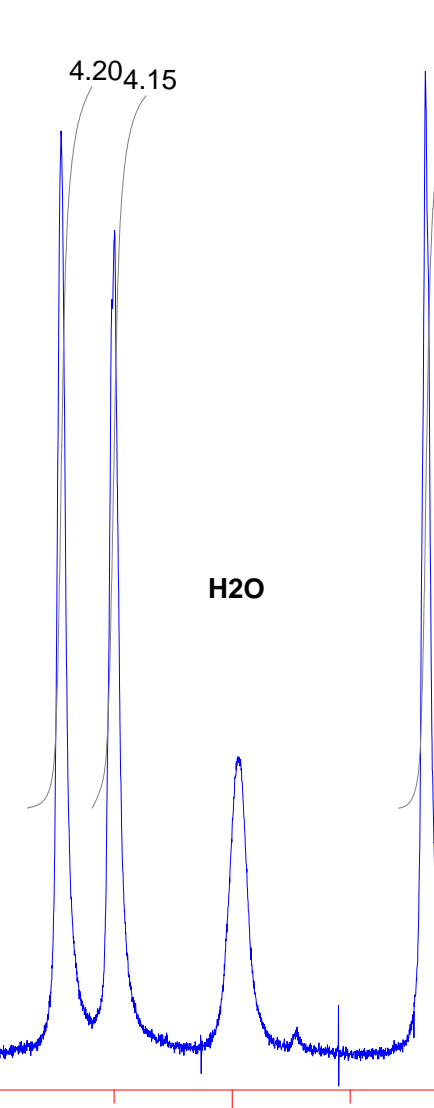

4
4.43

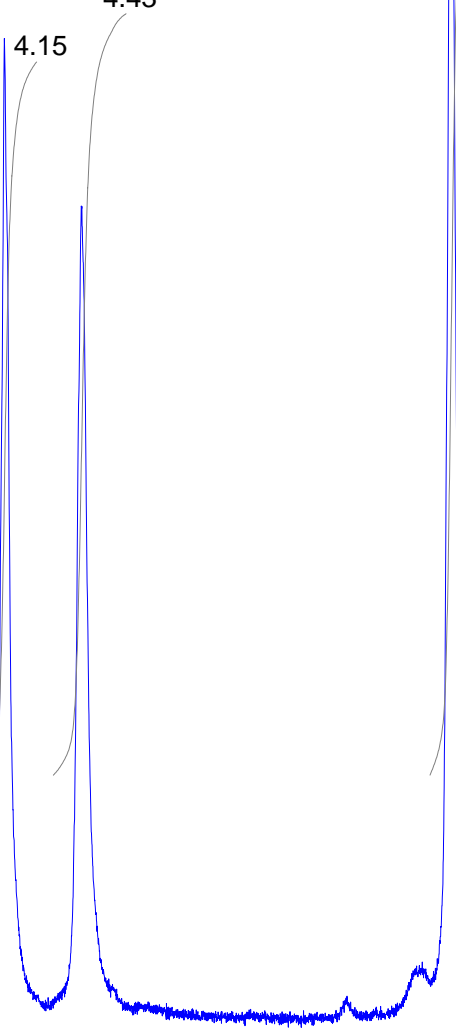

PPM

USER: -- DATE: Jul 262005 PTS1d: 16384

WinNuts - \$13bPd_100.fid 


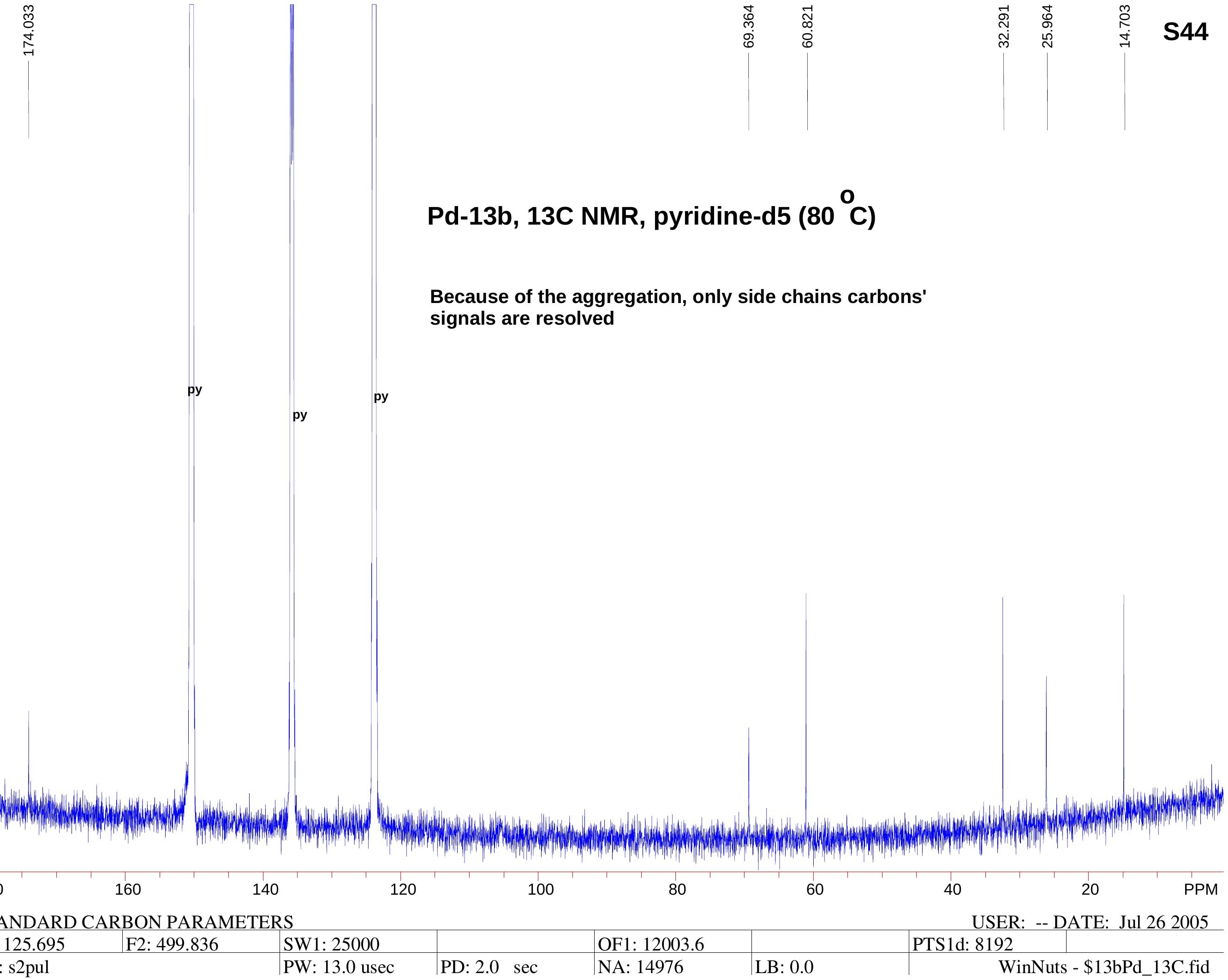

